\title{
Comprehensive Evaluation for Electric Power Information Security Based on Set Pair Analysis
}

\author{
Shouming Ma \\ School of Networks \& Telecommunications Engineering, Jinling Institute of Technology, Nanjing, P. R. China
}

\begin{abstract}
- the safe and stable operation of the information system has become the key content of the information works in the electric power industry. In order to establish a unified enterpriselevel security evaluation system, a new comprehensive evaluation method based on set pair analysis (SPA) was proposed utilizing its advantages of solving the uncertainty. The case results show that this method is an available, efficient solution to the electric power information security evaluation problem.
\end{abstract}

Keywords-electric power information security; set pair analysis; analytic hierarchy process; comprehensive evaluation

\section{INTRODUCTION}

Information techniques are gradually promoting the electric power system to go into the digital electric power stage [1]. Power information system not only includes production, scheduling and control system in power generation, transmission, substation, distribution and supply processes, but also includes production, marketing and other management system. There are great differences in the deployment environment, system function and security requirement of a variety of information systems in power production. Nowadays, power information security has become the key factor to ensure the safe and stable operation of power system [2-3]. In the development of information security protection, it is needed to build a complete set of information security evaluation index system, and use particular scientific methods to evaluate the system security level effectively.

The security evaluation of power information system can be considered as a multi-attribute decision-making problem. It is essentially a kind of uncertainty decision problem, which has a direct impact on the stability of the results. Set Pair Analysis (SPA) is a new mathematical theory and also a system analysis method of solving the uncertainty. The basic idea of SPA is to take the certainty and uncertainty into one system. Then the certainty and uncertainty of the research objects can be studied from three aspects, namely the identity degree, discrepancy degree and contrary degree [4-6]. The SPA is a simple and effective method to determine the relationship between the set pair and the uncertainty, which naturally provides a new idea for the security evaluation of power information system.

\section{SPA THEORY}

The SPA theory proposed by Keqin Zhao is a modified uncertainty theory which considering both certainties and uncertainties as an integrated system and depicting the certainty and uncertainty systematically from three aspects as identity, discrepancy and contrary [7-8]. Set pair refers to a couple that consists of two interrelated sets. The basic idea of SPA is to analyze the features of a set pair and set up a connection degree formula of these two sets including identity degree, discrepancy degree and contrary degree under certain circumstances. Based on the connection degree formula, a series of SPA-based researches have been conducted.

Putting together set A and B to form set pair $\mathrm{H}$ with respect to the problem $\mathrm{W}$, give the definition of the connection degree of set pair $\mathrm{H}$ as:

$$
\mu(W)=\frac{S}{N}+\frac{F}{N} i+\frac{P}{N} j
$$

where $\mu$ is the connection degree of the set pair, $N$ denotes the total number of characteristics of the set pair, $S, P$, and $F$ respectively represents the number of identity, contrary and discrepancy characteristics, $S / N, F / N$ and $P / N$ respectively represent the identity degree, discrepancy degree and contrary degree. $j$ is the coefficient of the contrary degree and was specified as -1 . As the coefficient of the discrepancy degree, $i$ is an uncertain value between -1 and 1 in terms of various circumstances.

Given $a=S / N, b=F / N, c=P / N$, (1) can be rewritten as follows:

$$
\mu=a+b i+c j
$$

As a result, the interactions, influences and transformations of the research objects can be represented fully. Due to its dialectic characteristics and advantages as a simple mathematic depiction with clear physical meaning, SPA has been applied to multi-attribute evaluation fields including modern management, applied math, computer science, engineering, social science, etc.

\section{MULTI-ATTRIBUTE EVALUATION BASED ON SPA}

We define the multi-attritute decision-making problem as $Q=\{S, E, W, D\} . S=\left\{s_{k}\right\}(k=1,2, \ldots, m)$ is the scheme set. $E=\left\{e_{r}\right\}(r=1,2, \ldots, n)$ is the indices set and $e_{r}$ represents the $r t h$ index. The positive index that express better situation with lager index value is marked as $E_{1}$, while the negative one $E_{2}$. $W=\left\{w_{1}, w_{2}, \ldots, w_{n}\right\}$ is the weight of index $e_{r} . D=\left\{d_{k r}\right\}$ represents the decision-making matrix about geiven problem $Q$ 
based on SPA, and $d_{k r}$ is the attribute value of index $e_{r}$ in the scheme $\mathrm{s}_{k}$.

The optimal evaluation set is marked as $U=\left\{u_{1}, u_{2}, \ldots, u_{n}\right\}$, while the worst evaluation set is marked as $V=\left\{v_{1}, v_{2}, \ldots, v_{n}\right\}$. $u_{r}$ and $v_{r}$ express the best and worst value of the index $e_{r}$. In the comparison space $[\mathrm{V}, \mathrm{U}]$, the identity degree of the pairs $\left\{d_{k r}, u_{r}\right\}$ and $\left\{d_{k r}, v_{r}\right\}$ in the domain $X r=\left\{d_{k r}, u_{r}, v_{r}\right\}$ is $a_{k r}$, the discrepancy degree is $c_{k r}$ and the contrary degree is $b_{k r}$. If $e_{r} \in E_{l}$, we can define the identity degree, discrepancy degree and contrary degree of $\left\{d_{k r}, u_{r}\right\}$ as (3), (4) and (5):

$$
\begin{gathered}
a_{k r}=\frac{d_{k r}}{u_{r}+v_{r}} \\
c_{k r}=\frac{d_{k r}^{-1}}{u_{r}^{-1}+v_{r}^{-1}}=\frac{u_{r} v_{r}}{\left(u_{r}+v_{r}\right) d_{k r}} \\
b_{k r}=1-a_{k r}-c_{k r}=\frac{\left(u_{r}-d_{k r}\right)\left(d_{k r}-v_{r}\right)}{\left(u_{r}+v_{r}\right) d_{k r}} \\
\text { So, the contact degree of set pair }\left\{d_{k r}, u_{r}\right\} \text { is: } \\
\mu\left\{d_{k r}, u_{r}\right\}=\frac{d_{k r}}{u_{r}+v_{r}}+\frac{\left(u_{r}-d_{k r}\right)\left(d_{k r}-v_{r}\right)}{\left(u_{r}+v_{r}\right) d_{k r}} i \\
+\frac{u_{r} v_{r}}{\left(u_{r}+v_{r}\right) d_{k r}} j
\end{gathered}
$$

Similarly, the contact degree of set pair $\left\{d_{k r}, v_{r}\right\}$ is:

$$
\begin{aligned}
& \mu\left\{d_{k r}, v_{r}\right\}=\frac{u_{r} v_{r}}{\left(u_{r}+v_{r}\right) d_{k r}}+\frac{\left(u_{r}-d_{k r}\right)\left(d_{k r}-v_{r}\right)}{\left(u_{r}+v_{r}\right) d_{k r}} i \\
& +\frac{d_{k r}}{u_{r}+v_{r}} j
\end{aligned}
$$

We can calculate the connect degree of the pair set $\left\{\mathrm{s}_{\mathrm{k}}, \mathrm{u}\right\}$ in comparison space $[\mathrm{U}, \mathrm{V}]$ :

$$
\mu\left\{s_{k}, u\right\}=a_{k}+b_{k} i+c_{k} j
$$

where, $a_{k}=\sum_{r=1}^{n} w_{r} a_{k r}, b_{k}=\sum_{r=1}^{n} w_{r} b_{k r}, c_{k}=\sum_{r=1}^{n} w_{r} c_{k r} \cdot a_{k r}$ and $c_{k r}$ are relative certainty and correspond to the affirmative and negative degree of how much $s_{k}$ close to the most optimal design, so under the relatively certain conditions, the relative closeness of $s_{k}$ and $u$ can be define as:

$$
r_{k}=\frac{a_{k}}{a_{k}+c_{k}}
$$

Obviously, the greater the $r_{k}$ is, the more the $s_{k}$ close to the optimal evaluation set. So, it can be used to sort a variety of schemes in accordance with the $r_{k}$ so as to select the most appropriate solution in those concrete practical applications.

\section{APPLICATION OF SPA IN THE POWER INFORMATION SECURITY}

\section{A. Comprehensive Evaluation Method Using SPA}

According to the particular characteristics of electric power information system, the steps of the SPA-based comprehensive evaluation method can be represented as follows.

a) According to the actual application situation, first of all, we must establish a clear evaluation index hierarchy of power information system.

b) Secondly, we need to construct a decision matrix, then calculating the relative closeness degree of each index.

c) Thirdly, the weight of each index was determined by the analytic hierarchy process (AHP).

d) Then, we calculate the comprehensive closeness degree of each scheme.

e) Finally, according to the comprehensive closeness degree, we can sort all electric power information system schemes. Obviously, those schemes with higher closeness degree are better than those with lower closeness degree.

\section{B. Case Study}

Taking into account the management and operation mode of electric power enterprises and their special protection objects, a unified information security evaluation and supervision mechanism is expected to be effectively established. A unified information security evaluation standard and institution is also needed to be formulated so as to form a complete and rigorous information security evaluation theory and method. Multi-attribute decision-making method based on set pair analysis can be used to solve the power information security evaluation.

In order to explain the method of this paper, based on the four indexes of the safety technology of the electric power information system, namely, physical security, network security, host security, application security, the existing three systems are evaluated. Clearly, these four indicators are efficiency indicators, the specific indicator values as shown in table 1 . The weights of the four indexes are $0.25,0.22,0.28$ and 0.25 . 
TABLE I. SCHEME INDEX VALUE

\begin{tabular}{|c|c|c|c|c|}
\hline \multirow{2}{*}{ Scheme } & \multicolumn{4}{|c|}{ Index } \\
\cline { 2 - 5 } & $\boldsymbol{e}_{\boldsymbol{1}}$ & $\boldsymbol{e}_{\mathbf{2}}$ & $\boldsymbol{e}_{\boldsymbol{3}}$ & $\boldsymbol{e}_{\boldsymbol{4}}$ \\
\hline $\boldsymbol{s}_{\boldsymbol{1}}$ & 62 & 77 & 99 & 32 \\
\hline $\boldsymbol{s}_{\boldsymbol{2}}$ & 61 & 71 & 100 & 23 \\
\hline $\boldsymbol{s}_{\boldsymbol{3}}$ & 64 & 73 & 96 & 30 \\
\hline
\end{tabular}

According to the above comprehensive evaluation algorithm using SPA, we can calculate the relative closeness degree of each scheme to the optimal scheme. As shown in table 2, the sorting result of these relative closeness degree is $r 1>r 3>r 2$. So, in these three schemes, according to the results, we can see the scheme 1 is the best scheme.

TABLE II. RELATIVE CLOSENESS VALUE

\begin{tabular}{|c|c|c|c|c|}
\hline \multirow{2}{*}{ Scheme } & \multicolumn{4}{|c|}{ Results } \\
\cline { 2 - 5 } & $\boldsymbol{a}_{\boldsymbol{k}}$ & $\boldsymbol{b}_{\boldsymbol{k}}$ & $\boldsymbol{c}_{\boldsymbol{k}}$ & $\boldsymbol{r}_{\boldsymbol{k}}$ \\
\hline $\boldsymbol{s}_{\boldsymbol{1}}$ & 0.525 & 0.002 & 0.473 & 0.53 \\
\hline $\boldsymbol{s}_{\boldsymbol{2}}$ & 0.474 & 0.002 & 0.524 & 0.48 \\
\hline $\boldsymbol{s}_{\boldsymbol{3}}$ & 0.508 & 0.003 & 0.489 & 0.51 \\
\hline
\end{tabular}

\section{CONCLUSIONS}

In order to realize the goal of information security of electric power enterprises, it is necessary to establish a set of advanced, practical and efficient information security assurance system. Among them, electric power information security assessment is a complex process of multi-level and multi-objective. The paper makes a comprehensive assessment of the security level by using the relative closeness of the set pair analysis, and has the advantages of clear thinking and simple and practical method. Our subsequent research should consider the multi-stage characteristics of security evaluation, and introduce the time series to make the set pair analysis method dynamic.

\section{ACKNOWLEDGMENT}

This work was supported by the research fund for advanced talents of JIT (Grants No. jit-b-201528) and the information \& communication engineering open project of JIT (Grants No. JIT-XTXK-201501).

\section{REFERENCES}

[1] Y. Zhu, B. Wang, S. Zhang, "The analysis and design of network and information security of electric power system," Transmission and Distribution Conference and Exhibition: Asia and Pacific, 2005 IEEE/PES. IEEE, 2005, pp.1-6.

[2] Y. Wang, S. Deng, W. Lin, T. Zhang, Y. Yu, "Research of electric power information security protection on cloud security,” Power System Technology (POWERCON), 2010 International Conference on. IEEE, 2010, pp.1-6.

[3] H. Tang, B. Mcmillin, "Security of information flow in the electric power grid,” Critical Infrastructure Protection, 2007, vol. 253, pp.43-56.

[4] Z. Zhou, "Decision support system based on set pair analysis and its application,” Engineering Sciences, 2007, vol. 03, pp.76-81.

[5] C. Liu, L. Zhang, A. Yang, S. Zhao, D. Li, "The evaluation model of international science and technology cooperation based on set pair analysis,” Journal of Interdisciplinary Mathematics, 2014, vol. 17, no. 1, pp.95-108.

[6] L. Li, Y. Cheng, L. Xie, L. Jiang, N. Ma, M. Lu, “An integrated method of set pair analysis and association rule for fault diagnosis of power transformers,” IEEE Transactions on Dielectrics \& Electrical Insulation, 2015, vol. 22, no. 4, pp.2368-2378.

[7] Y. Jiang, C. Xu, Y. Liu, K. Zhao, “A new approach for representing and processing uncertainty knowledge," Information Reuse and Integration, 2003. IRI 2003. IEEE International Conference on. IEEE, 2003, pp.466470 .

[8] K. Zhao, Y. Jiang, "Primary discussion on some systematical and dialectical thinking in set pair analysis,” Journal of Systemic Dialectics, 2000 . 